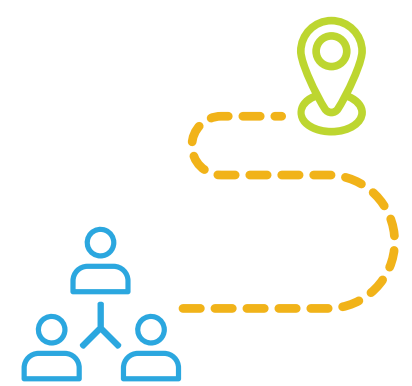

\title{
TRAJETÓRIA DA ARTICULAÇÃO DO "PROJETO QUALIREDE" COM A RESPOSTA PARA A SÍFILIS NO MUNICÍPIO DE CUIABÁ - MATO GROSSO, 2019
}

\author{
TRAJECTORY OF THE ARTICULATION OF THE "PROJECT QUALIREDE" WITH THE \\ RESPONSE FOR SYPHILIS IN THE MUNICIPALITY OF CUIABÁ - MATO GROSSO, 2019
}

\begin{abstract}
Míriam Estela de Souza Freire
Graduação em Enfermagem Obstetrícia pela UFMT (1992), com Especialização em Saúde Pública, Epidemiologia, Docência na Área de Vigilância da Saúde, Vigilância em Saúde de Doenças Transmissíveis, Mestranda em Saúde Coletiva, Servidora da Secretaria de Estadual de Saúde de Mato Grosso e Apoiadora Projeto "Sífilis Não" em Cuiabá - MT/MS/UFRN. E-mail: miriam.freire@lais.huol.ufrn.br.

Susi Astolfo

Graduação em Educação Física e Saúde Coletiva (UFMT). Especialista em Avaliação em Saúde e Gestão Federal do SUS, Mestra e doutoranda em Saúde Coletiva, Servidora na Superintendência Estadual do Ministério da Saúde em Mato Grosso (Seção de Apoio Institucional e Articulação Federativa). E-mail: susiastolfo@yahoo.com.br.

Ruth Terezinha Kehrig

Professora Associada e Pesquisadora do Programa de Pós-Graduação em Saúde Coletiva da Universidade Federal de Mato Grosso (UFMT). Doutora em Saúde Pública pela Universidade de São Paulo (2001), Administradora, Sanitarista e Gestora Pública atuando na área de Políticas, Planejamento, Gestão e Avaliação de Serviços no campo da Saúde Coletiva. ruth.kehrig@gmail.com.
\end{abstract}

\section{Fabiano Lima da Silva}

Graduação em Tecnologia em Processamento de Dados pela FIC e Direito pela UNIC, Especialista em Saúde Pública, Gestão do Trabalho e Educação em Saúde, Mestre em Saúde Coletiva, Professor da Universidade de Cuiabá (UNIC), Servidor da Secretaria Estadual de Saúde de Mato Grosso (SES-MT) e Pesquisador do Projeto QualiRede do Instituto de Saúde Coletiva (ISC - UFMT). E-mail: fabianolimsil@gmail.com.

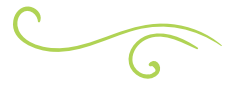

\section{RESUMO}

Diagnóstico oportuno, tratamento adequado e fortalecimento do contínuo do cuidado no enfrentamento da sífilis permitem reduzir a sua transmissão nos diversos grupos populacionais. A qualificação do cuidado em Infecções Sexualmente Transmissíveis, com destaque para a sífilis, propicia o controle desses agravos. Tal efeito depende, em parte, da qualidade da assistência ambulatorial e implica a articulação em rede entre os serviços especializados e de Atenção Primária à Saúde para o cuidado devido à saúde sexual e reprodutiva da população. 
A articulação de iniciativas relacionadas à qualificação do cuidado das Infecções Sexualmente Transmitidas, a exemplo do Projeto "Sífilis Não", assim como o "Projeto QualiRede", propõe uma intervenção com a finalidade de aprimorar o contínuo do cuidado a pessoas que vivem com agravos que compõem as Infecções Sexualmente Transmissíveis. No município de Cuiabá/MT, foi possível desenhar um projeto piloto de aproximação às intervenções preconizadas nos respectivos protocolos oficiais no âmbito do Sistema Único de Saúde. A metodologia de intervenção toma por base oficinas de aprendizado com responsáveis técnicos e coordenadores de Unidades Básicas de Saúde selecionados da Secretaria Municipal de Saúde de Cuiabá, com os objetivos de diagnóstico da situação e construção de planos de trabalho locais para o enfrentamento desses agravos. A intervenção operada consiste no automonitoramento local das ações por Unidades Básicas de Saúde, dados e informações das unidades, de indicadores de base populacional, de resultados clínicos e da qualidade organizacional dos serviços. A construção do contínuo do cuidado em Infecções Sexualmente Transmissíveis implica a integração das ações de promoção, prevenção, diagnóstico e assistência às Infecções Sexualmente Transmissíveis, com prioridade para a sífilis como opção institucional, a partir das unidades básicas de saúde. O presente relato objetiva mostrar os resultados da ação realizada até o momento.

Palavras-chave: Sífilis; Atenção Primária à Saúde; Qualidade da Assistência à Saúde.

\footnotetext{
ABSTRACT

The timely diagnosis, adequate treatment and strengthening of the continuum of care in the fight against syphilis allows to reduce its transmission in different population groups. The qualification of care in sexually transmitted infections, with emphasis on syphilis, provides control of these injuries. This effect depends in part on the quality of outpatient care and
}

implies the networking between specialized services and primary health care for care due to the population's Sexual and Reproductive Health. The articulation of initiatives related to the qualification of sexually transmitted infections care, like the "Project Siffilis Não", as well as the "QualiRede Project", proposes an intervention with the purpose of improving the continuum of care for people living with health problems that make up sexually transmitted infections. In the municipality of Cuiabá - MT, it was possible to design a pilot project to approximate the interventions recommended in the respective official protocols within the scope of Health Unic System. The intervention methodology was based on learning workshops with technical managers and coordinators of basic health units selected from the Municipal Health Secretariat of Cuiabá with the objectives of diagnosing the situation and building local work plans to face these grievances. The intervention operated was anchored in the local self-monitoring of actions by basic health units, data and information from the units, population-based indicators, clinical results and the organizational quality of services. The construction of the continuum of care in sexually transmitted infections implies the integration of actions to promote, prevent, diagnose and assist sexually transmitted infections, with priority for syphilis as an institutional option, based on basic health units. The present report aims to show the results of the action carried out so far.

Key-words: Syphilis; Primary Health Care; Quality of Health Care.

\section{INTRODUÇÃO}

A persistência da sífilis como um problema de saúde pública pode estar relacionada à falta de percepção dos profissionais de saúde de que a sífilis adquirida, em quaisquer de suas formas, com destaque para a sífilis na gestação e congénita, podem gerar 
graves consequências para mulher e seu concepto, tendo como fatores associados a ausência ou a baixa cobertura dos programas de prevenção, a existência de barreiras para o acesso à assistência pré-natal e a estigmatização com as infecções de transmissão sexual (GALBAN; BENZAKEN, 2007).

$\bigcirc$ aumento da incidência da sífilis congênita no Brasil e em outros países evidencia falhas na assistência pré-natal relacionadas ao controle de uma infecção, cujo Protocolo Clínico de Diretrizes Terapêuticas (PCDT) é bem divulgado, com triagem sorológica e tratamento de baixo custo. Esse fato contrasta com a redução da transmissão vertical do HIV, já demostrada no Brasil, apesar de possuir protocolos clínicos bem mais complexos e de custo elevado comparando com a transmissão vertical da sífilis, entretanto, é mais consolidado entre os profissionais e serviços de saúde (AMARAL, 2012).

No período de 2016 a 2019, a Organização Mundial da Saúde (OMS) divulgou que a sífilis atingiu mais de 12 milhões de pessoas em todo o mundo, calculou-se um total de 6,3 milhões de casos de sífilis curáveis (WHO, 2019). No Brasil, em 2018, foram notificados cerca de 158 mil casos de sífilis adquirida, 63 mil casos de sífilis em gestantes e, aproximadamente, 26 mil casos de sífilis congênita. Esses números apontam aumento de $28,3 \%$ e $25,7 \%$ na taxa de detecção de sífilis adquirida e de sífilis em gestantes respectivamente, e de 5,2\% na incidência de sífilis congênita no comparativo de 2017 para 2018 (BRASIL, 2020a).

O Ministério da Saúde (BRASIL, 2020b), por intermédio do então Departamento de IST, HIV/aids e hepatites virais, preocupado com o cenário epidemiológico da sífilis, decretou epidemia de sífilis no Brasil em 2016. Isso exigiu das autoridades sanitárias, nos diferentes entes federados, ações estratégicas com vista à redução dos números da sífilis no país. No início de 2017, foi aprobada, por meio da Lei Orçamentária Anual No 13.414, a incorporação de recursos para utilização em ações de enfrentamento ao agravo (BRASIL, 2020b).
Segundo o Boletim Epidemiológico de Sífilis de 2020, o Brasil, assim como muitos países, apresenta uma reemergência da sífilis, com o aumento da taxa de detecção para sífilis adquirida saindo de 2,1 em 2010 para 44,5 em 2016. Diante disso, os profissionais de saúde devem estar aptos a reconhecer as manifestações clínicas, conhecer os testes diagnósticos disponíveis, principalmente interpretar o resultado do exame para diagnóstico e controle de tratamento (BRASIL, 2020b).

Em 2017, o Ministério da Saúde convidou os gestores estaduais e municipais para aderirem à estratégia nacional de enfrentamento à sífilis por meio da "Pesquisa aplicada para integração inteligente orientada ao fortalecimento das redes de atenção para resposta rápida à sífilis", conhecido como Projeto "Sífilis Não", que tem por objetivo geral contribuir com a redução da sífilis adquirida e em gestantes, além da eliminação da sífilis congênita no Brasil (BRASIL, 2020a). As ações estão focadas na ampliação do acesso ao diagnóstico precoce; prescrição e administração da penicilina; e organização da linha de cuidado para sífilis nas unidades de APS, com destaque para a ação de investigação e seguimento dos casos de sífilis em gestante e sífilis congênita nos municípios prioritários do Projeto "Sífilis Não" (BRASIL, 2020a).

O QualiRede, por sua vez, tem como proposta um projeto de intervenção com a finalidade de aprimorar a atenção integral em infecção sexualmente transmissível por meio da qualificação da rede de cuidados (NEMES et al., 2019). Com essa inspiração, realizou-se um projeto piloto do seu desenvolvimento para contribuir com o controle da sífilis em Cuiabá, onde foram realizadas articulações de pesquisadores do Instituto de Saúde Coletiva da Universidade Federal de Mato Grosso (UFMT) com as áreas técnicas da APS, Vigilância Epidemiológica, Serviço de Assistência Especializada (SAE), Saúde da Mulher e Rede Cegonha da SMS de Cuiabá. Nesse contexto, este relato apresenta a trajetória do projeto piloto do QualiRede no município de Cuiabá. 


\section{MÉTODO}

O modelo QualiRede do contínuo do cuidado em HIV/aids, hepatites virais e outras IST/sífilis contempla os seguintes momentos e finalidades (NEMES et al., 2016):

- Promoção da saúde sexual e reprodutiva (SSR): estimular a autonomia e o bem-estar na vivência da sexualidade e da reprodução;

- Prevenção combinada: evitar contato com os agentes infecciosos e, quando houver contato, impedir que resulte em infecção;

- Diagnóstico: precoce e oportuno;

- Vinculação: ao seguimento de saúde integral;

- Tratamento: oferecer tratamento efetivo para todos os infectados;

- Retenção: manter todos os pacientes em seguimento de saúde;

- Adesão: incentivar, monitorar e apoiar o seguimento do tratamento;

- Supressão: obter bom desfecho clínico e diminuição da transmissão na comunidade.

Considerando a estrutura proposta pelo projeto, segue o relato das atividades realizadas no período de execução das duas oficinas no município de Cuiabá: 1) iniciativas de preparação e abertura do projeto; 2) reuniões de constituição do grupo de pesquisa com técnicos-chave dos serviços envolvidos; 3) movimentos de articulação do projeto de pesquisa com serviços do SUS (gestão estadual - SES/MT e gestores de secretarias municipais de saúde); 4) instituição do Grupo de Trabalho Piloto de Cuiabá (GT-SAE e GT-APS); 5) ações preparatórias e a realização da Oficina I Piloto de diagnóstico da situação do contínuo do cuidado em IST nas unidades participantes; 6) atividades preparatórias e a realização da Oficina II Piloto de análise da situação/seguimento de casos de sífilis por UBS Piloto; 8) Monitoramento e avaliação.
A intervenção por UBS piloto se resume a quatro momentos, como segue:

O Momento 1 compõe a construção da cascata epidemiológica do contínuo do cuidado em sífilis (população estimada com sífilis, população diagnosticada, tratada adequadamente e curada), com uso dos dados epidemiológicos dos registros da própria unidade, além de dados epidemiológicos dos sistemas de informação do Sistema Único de Saúde (SUS) das unidades participantes. A equipe de técnicos e pesquisadores QualiRede sintetizou uma planilha de seguimento da sífilis para o serviço acompanhar sua coorte de pacientes, com dados do cotidiano do serviço, a partir do prontuário eletrônico, ou por planilha Excel, ou livros de registro e fichas com planilhas manuais. Dessa planilha, foram extraídos os dados para a cascata (população tratada e curada), a população diagnosticada foi extraída do SINAN e a população com sífilis foi estimada (diagnosticados $+30 \%$ de subnotificação). Além disso, essa planilha serviu de base para o levantamento dos problemas na primeira oficina, como consta do Momento 3.

Para o Momento 2, foram utilizadas informações do banco de dados de aplicação anterior, do questionário QualiAB-SSR, em 2017, no município e da sua nova aplicação manualmente pelas próprias unidades selecionadas, interessadas em dispor dessa avaliação mais atualizada. Os resultados dessas aplicações, registrados em bancos de dados de planilha do Excel, informam sobre o desempenho das UBS em saúde sexual e reprodutiva, os quais são relacionados com as etapas do contínuo do cuidado. Em projeto de dissertação da apoiadora do MS para o Projeto "Sífilis Não", serão levantados dados das unidades participantes do projeto antes (2019) e após (2021) a intervenção nos dois primeiros momentos: situação epidemiológica (cascata e planilha) e avaliação QualiAB-SSR.

O Momento 3 é divido em duas etapas (oficinas). Na primeira, ocorreu o levantamento dos 
principais problemas das unidades no cuidado às pessoas com sífilis. Na segunda oficina, são construídos os planos de ações de cada unidade para o monitoramento do contínuo do cuidado e enfrentamento dos problemas.

Em relação ao Momento 4, que se refere à avaliação do modelo de intervenção aplicado, ocorrerá a avaliação das ações realizadas, dos dados das unidades (planiIha de seguimento) e dos indicadores do QualiAB-SSR levantados após as oficinas em comparação aos mesmos dados coletados anteriormente às oficinas. As UBS participantes desse projeto piloto foram doze (12): Novo Colorado II, Dr. Fábio II, Tijucal, Pico do Amor, Liberdade, CPA IV, Três Barras, Quilombo, Alvorada, Clínica da Família CPA I, Santa Teresinha e Jóquei Club. Até o presente momento não foi realizado o Momento 4 (monitoramento e avaliação) deste projeto piloto, que ainda está em andamento.

\section{RESULTADOS E LIÇÕES APRENDIDAS}

O aumento de cobertura da testagem para HIV, hepatites virais e sífilis, entre outras IST, implica sua priorização na APS e em outros serviços de porta de entrada ao SUS, o que certamente aumentará o número de pessoas com diagnóstico positivo e a desejável cobertura assistencial, que propicie o tratamento e a qualidade necessários em todo o contínuo do cuidado do paciente. A construção das cascatas do contínuo do cuidado em sífilis nas 12 unidades piloto mostrou que havia falhas em todas as etapas do contínuo, desde o diagnóstico até a supressão da sífilis em Cuiabá. Quanto ao questionário QualiAB-SSR, sua aplicação apontou a necessidade das UBS em rever a organização das ações do contínuo do cuidado em sífilis. A planilha de seguimento ofertada pelo projeto propiciou otimização e organização do processo de trabalho nas UBS, bem como melhoria no seguimento dos casos de sífilis nessas unidades.
Cabe destacar que o aprendizado de novas práticas, a partir da reflexão sobre as próprias práticas, deve ser desenvolvido de forma contínua, o que propicia a melhoria do contínuo do cuidado. Isso se dá por meio da educação permanente e de oferta de novas metodologias de trabalho.

\section{CONSIDERAÇÕES FINAIS}

As ações e estratégias de enfrentamento à sífilis em Cuiabá mobilizaram os profissionais de saúde da APS, Vigilância à Saúde, Saúde da Mulher, Rede Cegonha, SAE, UFMT, área técnica das coordenações de IST municipal que empenharam esforços em difundir as ações de enfrentamento à sífilis. A capilaridade e a potência do Projeto já foram observadas na melhoria da cobertura da testagem rápida, dispensação e administração da penicilina na rede de APS e no manejo da sífilis na própria APS, sem necessidade de encaminhamento para uma referência secundária para seu manejo nas próprias unidades participantes do projeto piloto.

As novas tecnologias e metodologias disponibilizadas aos profissionais de saúde da rede APS do município de Cuiabá propiciaram novas estratégias e possibilidades no controle e enfrentamento à sífilis e outras IST. O monitoramento das ações prestadas nessas etapas, a partir do diagnóstico realizado nos próprios serviços, com intervenção sobre problemas prioritários no contínuo do cuidado, assim como a articulação em linhas de atenção, são essenciais para o enfrentamento desse conjunto de agravos.

\section{AGRADECIMENTOS}

Agradeço aos coordenadores e pesquisadores do Projeto "Sífilis Não" pelas orientações, em especial, ao supervisor Adriano Santiago dos Santos, minha gratidão, pelo incentivo e apoio nesta caminhada. 
À Professora Doutora, Maria Inês Battistella Nemes, da Universidade de São Paulo (USP), pela oportunidade de conhecermos o Projeto QualiRede, que nos guiou na qualificação do contínuo do cuidado nas IST.

À coautora, Professora Doutora Ruth Terezinha Kehrig, do Instituto de Saúde Coletiva (ISC - UFMT), por acreditar nas potencialidades regionais e incluir o município de Cuiabá no Projeto QualiRede MT.

Agradecimento especial para área técnica de IST-aids e hepatites virais, Vigilância Epidemiológica e Atenção Primária da Secretaria Municipal de Saúde de Cuiabá, principalmente às enfermeiras coordenadoras das UBS Piloto, por todo o apoio técnico recebido nessa construção e confiança em desenvolver as ações ora descritas. Gratidão a todos! 


\section{REFERÊNCIAS}

AMARAL, E. Sífilis na gravidez e óbito fetal: de volta para o futuro. Rev. Bras. Ginecol. Obstet, Rio de Janeiro, v. 34, n. 2, p. 52-55, fev. 2012.

BRASIL. Lei $\mathbf{n}^{\circ} \mathbf{1 3 . 4 1 4}$, de 10 de janeiro de 2017. Estima a receita e fixa a despesa da União para o exercício financeiro de 2017. Disponível em: http://www.planalto.gov.br/ ccivil_03/_Ato2015-2018/2017/Lei/L13414.htm\#: :text=Estima\%20a\%20receita\%20e\%20 fixa,cargo\%20de\%20PRESIDENTE\%20DA\%20REP\%C3\%9ABLICA. Acesso em: 18 mar. 2021.

BRASIL. Ministério da Saúde. Boletim Epidemiológico da Sífilis, Brasília, ano VI, n. especial, out. 2020a. Disponível em: http://www.aids.gov.br/system/tdf/pub/2016/67373/boletim_ sifilis_2020_especial.pdf?file=1\&type=node\&id=67373\&force=1. Acesso em: 12 nov. 2020.

BRASIL. Ministério da Saúde. Resposta rápida à sífilis: uma análise situacional da estratégia do apoio institucional. In: BRASIL. Ministério da Saúde. Boletim Epidemiológico. Brasília, v. 51, n. 42 , p. $17-21$, out. 2020 b.

BRASIL. Ministério da Saúde. Guia Política Nacional de Atenção Básica - Módulo 1 :

integração atenção básica e vigilância em saúde. Brasília: Ministério da Saúde, 2018. 68 p.

BRASIL. Ministério da Saúde. 5 passos para a implementação do manejo da infecção pelo HIV na Atenção Básica: guia para gestores. Brasília: Ministério da Saúde, 2014.

GALBAN, E.; BENZAKEN, A. S. Situación de la sífilis em 20 países de LatinoAmérica y el Caribe: año 2006. DST - J bras Doenças Sex Transm, v. 19. n. 3-4, p. 166-172, 2007.

NEMES, M. I. B. et al. QualiRede DST-HIV-HV: qualificação da Rede de Cuidados em DST, HIV/ aids e hepatites virais em regiões prioritárias dos estados de Santa Catarina e São Paulo. [S. n: s. I], 2016. (Projeto de Pesquisa - em andamento). Departamento de Medicina Preventiva, Faculdade de Medicina. São Paulo, USP. Disponível em: https://cutt.ly/LbNiEvO. Acesso em: 6 nov. 2020.

NEMES, M. I. B. et al. A intervenção QualiRede: melhoria do desempenho do contínuo do cuidado em HIV, sífilis congênita e hepatite $\mathrm{C}$ em regiões de saúde. Rev. Bras. Epidemiol. v. 22, supl. 1, p. 1-15, 2019.

ORGANIZAÇÃO MUNDIAL DE SAÚDE - OMS. Global prevalence and incidence of selected curable sexually transmitted infections overview and estimates. Geneva: WHO, 2019. 52 p. Disponível em https://www.who.int/hiv/pub/sti/pub7/en/: Acesso em: 5 nov. 2020.

UNIVERSIDADE FEDERAL DO RIO GRANDE DO NORTE - UFRN. Laboratório de Inovação Tecnológica em Saúde - LAIS. Secretaria de Educação a Distância - SEDIS. Plano de Trabalho do Projeto de Pesquisa aplicada para integração inteligente orientada ao fortalecimento das redes de atenção para resposta rápida à sífilis. Natal: UFRN, 2017. 65p.

WORLD HEALTH ORGANIZATION - WHO. Global health sector strategy on sexually transmitted infections, 2016-2021: Towards ending STIs. Geneva: WHO, 2016.

Disponível em: https://cutt.ly/XbNu4uw. Acesso em: 5 nov. 2020. 